Tohoku Math. J.

66 (2014), 355-375

\title{
DANILOV'S RESOLUTION AND REPRESENTATIONS OF THE MCKAY QUIVER
}

\author{
OSKAR KĘDZIERSKI
}

(Received January 11, 2011, revised June 21, 2013)

\begin{abstract}
We construct a family of McKay quiver representations on the Danilov resolution of the $\frac{1}{r}(1, a, r-a)$ singularity. This allows us to show that the resolution is the normalization of the coherent component of the fine moduli space of $\theta$-stable McKay quiver representations for a suitable stability condition $\theta$. We describe explicitly the corresponding union of chambers of stability conditions for any coprime numbers $r, a$.
\end{abstract}

1. Introduction. In [12] King introduced the notion of stability of a quiver representation and via GIT constructed the fine moduli of stable representations. A well-known example of King's moduli is the $G$-Hilbert scheme for any finite group $G \subset \operatorname{GL}(n, \boldsymbol{C})$, i.e., the scheme parameterizing all $G$-invariant 0-dimensional subschemes of $\boldsymbol{C}^{n}$ of length equal to the order of $G$. It is the moduli of representations of the McKay quiver, defined by the inclusion of $G$ in the general linear group and representation theory of $G$. The $G$-Hilbert scheme was introduced by Ito and Nakamura in [9], where they proved that it is the minimal (crepant) resolution of $\boldsymbol{C}^{2} / G$ for a finite group $G \subset \operatorname{SL}(2, C)$, and the relation of the $G$-Hilbert scheme with the moduli of representations of the McKay quiver was observed by Ito and Nakajima in [8]. The result from [9] was extended by Bridgeland, King and Reid [1] by showing that the $G$-Hilbert scheme is a crepant resolution of the singularity $C^{3} / G$ for any finite group $G \subset \operatorname{SL}(3, C)$. In particular, the moduli is smooth and irreducible. Moreover, if $G$ is abelian, it turns out that by varying the stability parameter one can get all projective crepant resolutions of $C^{3} / G$ (cf. [2]). The above results suggest that it may be possible to interpret some other resolutions of quotient singularities as moduli of the McKay quiver representations.

In the following paper we accomplish this task for the Danilov resolution of 3-dimensional cyclic terminal quotient singularities. The proof relies on Logvinenko's classification of all natural families (called gnat-families) of McKay quiver representations on some fixed resolution $Y$ of $\boldsymbol{C}^{n} / G$ for any finite abelian group $G \subset \mathrm{GL}(n, \boldsymbol{C})$ and his characterization of such families as satisfying the reductor condition [13]. The most obvious candidate to be isomorphic to the resolution is the coherent component defined by Craw, Maclagan and Thomas as the irreducible component of stable moduli of the McKay quiver representations containing points parameterizing free orbits. In [3], they prove that, for a finite abelian group

2010 Mathematics Subject Classification. Primary 14E16; Secondary 16G20, 14L24.

Key words and phrases. McKay correspondence, resolutions of terminal quotient singularities, Danilov resolution, moduli of quiver representations.

Research supported by a grant of Polish MNiSzW (N N201 2653 33). 
$G$, it is a possibly non-normal toric variety which admits projective birational morphism to $C^{n} / G$.

Let $G=\left\langle\operatorname{diag}\left(\varepsilon, \varepsilon^{a}, \varepsilon^{r-a}\right)\right\rangle$ be a cyclic group of order $r$ generated by a diagonal matrix, where $\varepsilon=e^{2 \pi i / r}$ for fixed coprime numbers $a, r$ such with $r>1$. The quotient singularity $X=C^{3} / G$ is a 3 -dimensional cyclic, terminal quotient singularity (cf. [14]). We call this the singularity of type $\frac{1}{r}(1, a, r-a)$. In his proof of the weak factorization theorem for toric threefolds, Danilov [4] introduced a recursively defined resolution of the singularity of type $\frac{1}{r}(1, a, r-a)$, which was subsequently named Economic resolution by Reid [15, p. 381].

For $a= \pm 1$, Kędzierski [11] proved that the Danilov resolution is isomorphic to the component of the $G$-Hilbert scheme that contains the free $G$-orbits. This cannot hold for $a \neq \pm 1$ as the $G$-Hilbert scheme is singular. Our main result Theorem 8.2 establishes that, for $a \neq \pm 1$, the Danilov resolution is isomorphic to the normalization of the coherent component of the moduli space of representations of the McKay quiver for a suitably chosen stability parameter. In fact, we describe precisely the appropriate union of GIT chambers of stability conditions. Finally, we conjecture that the coherent component for such stability conditions is normal.

The main idea is to define a priori a family of McKay quiver representations on the Danilov resolution and to use the universal property of the moduli space. To show that the resulting map is injective, we prove that any two representations in the given family are nonisomorphic. This is done by exploiting the recursive nature of the resolution. On the level of representations, the recursive step can be seen by grouping the vertices of the McKay quiver in so-called L-bricks and R-bricks. Those bricks can be seen as vertices of smaller McKay quivers.

The paper is organized as follows. In Section 2, we recall the definition of the Danilov resolution. In Section 3, we define effective divisors $X_{i}, Y_{i}, Z_{i}$ and $Q$-divisors $R_{i}$ which will be used in constructing a family of McKay quiver representations on the Danilov resolution. The McKay quiver is defined in Section 4. The family of quiver representations is constructed in Section 5 and we check that any two representations in that family are non-isomorphic. In Section 6, we recall elementary facts on stability of quiver representations. In Section 7, we determine a cone of stability conditions for the constructed family. Finally, in Section 8 we prove the main theorem, and compute explicitly the union of chambers of stability conditions for $\frac{1}{5}(1,2,3)$.

The author would like to thank Miles Reid for introducing him into this subject and to thank anonymous referee for his/her careful reading of the paper and for a great deal of effort put into its improvement. The author is grateful to Alastair Craw for helpful remarks and his help in checking the English grammar.

2. Recursive definition of the Danilov resolution. We denote $N_{0}=\boldsymbol{Z} e_{1} \oplus \mathbf{Z} e_{2} \oplus \mathbf{Z} e_{3}$ the free $\boldsymbol{Z}$-module with the basis $\left\{e_{1}, e_{2}, e_{3}\right\}$, and $M_{0}=\operatorname{Hom}_{\boldsymbol{Z}}\left(N_{0}, \boldsymbol{Z}\right)=\boldsymbol{Z} e_{1}^{*} \oplus \boldsymbol{Z} e_{2}^{*} \oplus \boldsymbol{Z} e_{3}^{*}$ with the dual basis $\left\{e_{1}^{*}, e_{2}^{*}, e_{3}^{*}\right\}$. The vector $a_{1} e_{1}+a_{2} e_{2}+a_{3} e_{3}$ is also denoted by $\left(a_{1}, a_{2}, a_{3}\right)$. 
For coprime integers $0 \leq a<r$, we denote by $G(r, a)$ the finite cyclic subgroup of $\operatorname{GL}(3, \boldsymbol{C})$ generated by the diagonal matrix $\operatorname{diag}\left(\varepsilon, \varepsilon^{a}, \varepsilon^{r-a}\right)$, where $\varepsilon=e^{2 \pi i / r}$.

We set

$$
N(r, a)=N_{0}+Z \frac{1}{r}\left(e_{1}+a e_{2}+(r-a) e_{3}\right)=N_{0}+Z(1 / r, a / r,(r-a) / r)
$$

and $M(r, a)=\operatorname{Hom}_{\boldsymbol{Z}}(N(r, a), \boldsymbol{Z})$. The lattice $M(r, a)$ is identified with the sublattice of $M_{0}$ consisting of the exponents of $G(r, a)$-invariant Laurent monomials.

For any elements $p_{1}, \ldots, p_{n}$ in $N(r, a)$, we denote by $\left\langle p_{1}, \ldots, p_{n}\right\rangle$ the cone generated by these points. For a rational polyhedral cone $\sigma$ in $N(r, a) \otimes_{\mathbf{Z}} \boldsymbol{R}$, we denote by $U_{\sigma}$ the toric $\operatorname{chart} \operatorname{Spec}\left(\boldsymbol{C}\left[\sigma^{\vee} \cap M(r, a)\right]\right)$.

For a positive integer $t$ and an integer $s$, we denote by $\langle s\rangle_{t}$ the least non-negative integer $u$ such that $t$ divides $s-u$. Sometimes, we write it simply $\langle s\rangle$ when $t$ is obvious.

Let $r$ and $a$ be coprime integers with $0 \leq a<r$. We recall the definition of the Danilov resolution of the singularity $\frac{1}{r}(1, a, r-a)$ (cf. [15, p. 381]). Let $\Delta(r, a)=\left\langle e_{1}, e_{2}, e_{3}\right\rangle$ be the positive octant in $N(r, a) \otimes_{Z} \boldsymbol{R}$. There exists a ring isomorphism of $\boldsymbol{C}\left[\Delta(r, a)^{\vee} \cap M(r, a)\right]$ with the ring of $G(r, a)$-invariant regular functions on $\boldsymbol{C}^{3}$. Therefore the quotient $X(r, a)=$ $C^{3} / G(r, a)$ is the toric variety given by the cone $\Delta(r, a)$ in the real vector space with underlying lattice $N(r, a)$. Note that $X(1,0)$ is a smooth variety and for $r \geq 2$ the quotient $X(r, a)$ is singular.

Let $b$ be the inverse of $a$ modulo $r$. Set

$$
p_{i}=\frac{1}{r}\left(\langle-i b\rangle_{r}, r-i, i\right) \in N(r, a) \text { for } i=0, \ldots, r .
$$

Note that $p_{r-a}=1 / r(1, a, r-a)$, which represents the type of the singularity and $p_{0}=e_{2}, p_{r}=e_{3}$. The following well-known lemma implies that the toric varieties associated to cones $\left\langle e_{1}, e_{2}, p_{r-a}\right\rangle,\left\langle e_{1}, e_{3}, p_{r-a}\right\rangle$ are isomorphic to the quotients of type $1 /(r-a)$ $\left(1,\langle r\rangle_{r-a},\langle-r\rangle_{r-a}\right)$ and $1 / a\left(1,\langle-r\rangle_{a},\langle r\rangle_{a}\right)$, respectively.

LEMMA 2.1. There exist $\mathbf{Z}$-linear isomorphisms

$$
\begin{gathered}
L(r, a): N\left(r-a,\langle r\rangle_{r-a}\right) \rightarrow N(r, a), \\
R(r, a): N\left(a,\langle-r\rangle_{a}\right) \rightarrow N(r, a),
\end{gathered}
$$

such that $L(r, a)\left(\Delta\left(r-a,\langle r\rangle_{r-a}\right)\right)=\left\langle e_{1}, e_{2}, p_{r-a}\right\rangle, R(r, a)\left(\Delta\left(a,\langle-r\rangle_{a}\right)\right)=\left\langle e_{1}, e_{3}, p_{r-a}\right\rangle$, and $L(r, a)\left(e_{1}\right)=R(r, a)\left(e_{1}\right)=e_{1}, L(r, a)\left(e_{3}\right)=R(r, a)\left(e_{2}\right)=p_{r-a}$.

DEFINITION 2.2. By a weighted blow-up of the singularity of type $\frac{1}{r}(1, a, r-a)$ at the point $p_{r-a}=1 / r(1, a, r-a)$, we mean a toric variety $\overline{X(r, a)}$ obtained by the star subdivision of the cone $\Delta(r, a)$ at the point $1 / r(1, a, r-a)$.

The weighted blow-up induces a proper, birational morphism $\overline{X(r, a)} \rightarrow X(r, a)$ with exceptional divisor equal to the weighted projective space $\boldsymbol{P}(1, a, r-a)$.

DEFinition 2.3. The Danilov resolution of the singularity of type $\frac{1}{r}(1, a, r-a)$ is a resolution obtained by the weighted blow-up of the singularity of type $\frac{1}{r}(1, a, r-a)$ at 


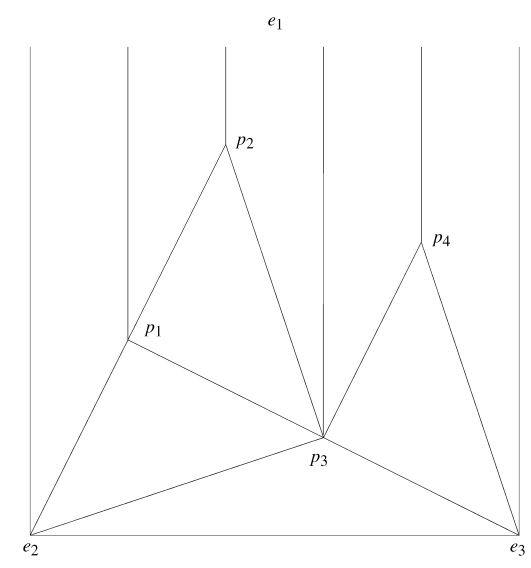

Figure 1. The fan of Danilov resolution of $\frac{1}{5}(1,2,3)$ cut with hyperplane $e_{2}^{*}+e_{3}^{*}=1$.

the point $p_{r-a}$ and, recursively, the Danilov resolutions of the singularities of type $1 /(r-a)\left(1,\langle r\rangle_{r-a},\langle-r\rangle_{r-a}\right)$ and $1 / a\left(1,\langle-r\rangle_{a},\langle r\rangle_{a}\right)$.

DEFINITION 2.4. For fixed $r$ and $a$, we call the resolution of $1 /(r-a)\left(1,\langle r\rangle_{r-a}\right.$, $\left.\langle-r\rangle_{r-a}\right)$ singularity an L-resolution and the resolution of $1 / a\left(1,\langle-r\rangle_{a},\langle r\rangle_{a}\right)$ an R-resolution. The 3-dimensional cones of the fan of the Danilov resolution will be called L-cones or $\mathrm{R}$-cones if they are subsets of the cones $\left\langle e_{1}, e_{2}, p_{r-a}\right\rangle$ or $\left\langle e_{1}, e_{3}, p_{r-a}\right\rangle$, respectively.

The fan of the Danilov resolution consists of $2 r-1$ simplicial cones of dimension three. Precisely $r$ cones of dimension three contain $e_{1}$. We denote them $\sigma_{0}, \ldots, \sigma_{r-1}$.

DEFINITION 2.5. Set

$$
\sigma_{i}=\left\langle p_{i}, p_{i+1}, e_{1}\right\rangle \text { for } i=0, \ldots, r-1 .
$$

Note that the resolution can be constructed by $r-1$ weighted blow-ups at the points $p_{1}, \ldots, p_{r-1}$ with a suitable order.

COROLLARY 2.6. The Danilov resolution of the singularity $\frac{1}{r}(1,1, r-1)$ is obtained by the consecutive blow-ups at the points $p_{r-1}, \ldots, p_{1}$. In the case of $\frac{1}{r}(1, r-1,1)$, the blow-ups are done at the points $p_{1}, \ldots, p_{r-1}$.

Definition 2.7. For fixed $r$ and $a$, let $D_{i}(r, a)$ denote the $T$-invariant toric divisor associated to the ray generated by the lattice point $p_{i}$ for $i=0, \ldots, r$. Let $E_{j}$ denote the $T$-invariant toric divisor associated to the ray generated by $e_{j}$ for $j=1,2,3$.

Note that $D_{0}(r, a)=E_{2}$ and $D_{r}(r, a)=E_{3}$. To simplify notation, we write $D_{i}$ instead of $D_{i}(r, a)$. 
3. Divisors $X_{i}, Y_{i}, Z_{i}, R_{i}$ and their properties. In this section we start by defining recursively a permutation $\tau(r, a)$ of $\{0,1, \ldots, r-1\}$. It will be subsequently used in the construction of divisors $X_{i}, Y_{i}, Z_{i}$ on the Danilov resolution. These divisors will define the structure of a gnat-family in the sense of Logvinenko [13] on the Danilov resolution.

Definition 3.1. If $a \in\{1, r-1\}$ set $\tau(r, a)(i)=\langle a i-1\rangle_{r}$ for $i=0, \ldots, r-1$, and otherwise

$$
\tau(r, a)(i)= \begin{cases}\tau\left(r-a,\langle r\rangle_{r-a}\right)\left(\langle i\rangle_{r-a}\right) & \text { if } i \geq a, \\ (r-a)+\tau\left(a,\langle-r\rangle_{a}\right)(i) & \text { if } i<a .\end{cases}
$$

Observe that $\tau(r, a)(0)=r-1$. Note the recursive nature of the above definition. The permutation $\tau$ will play a crucial role in determining the stability parameters connected with the moduli structure on the Danilov resolution.

EXAMPLE 3.2. In order to compute $\tau(5,2)$ we need to know $\tau(2,1)=(0,1)$ and $\tau(3,2)=(0,2)$ (written as cyclic permutations in the standard notation). Then,

$$
\begin{gathered}
\tau(5,2)(i)=\tau(3,2)\left(\langle i\rangle_{3}\right) \quad \text { for } i \geq 2, \\
\tau(5,2)(i)=3+\tau(2,1)(i) \quad \text { for } i<2,
\end{gathered}
$$

hence $\tau(5,2)=(0,4,1,3,2)$ as a cyclic permutation. In an analogous way, we could check that $\tau(7,2)=(0,6,3,2)(1,5,4)$ which in turn allows us to compute $\tau(12,7)=$ $(0,11,3,7)(1,10,4,6,8,2,5,9)$.

DEFINITION 3.3. We call the sequence of numbers $i, i+(r-a), \ldots, i+s(r-a)$ an L-brick if

i) $i<r-a$,

ii) $i+(s+1)(r-a)>r$,

iii) every number in the sequence is strictly smaller than $r$.

The sequence of numbers $i, i+a, \ldots, i+s a$ is called an R-brick if

1) $i<a$,

2) $i+(s+1) a>r$,

3) every number in the sequence is strictly smaller than $r$.

The L- and R-bricks connect the characters of a cyclic group of order $r$ with the characters of cyclic groups of order $r-a$ and $a$, respectively. They can be identified with fibers of the projections $\{0, \ldots, r-1\} \rightarrow \boldsymbol{Z} /(r-a) \boldsymbol{Z}$ and $\{0, \ldots, r-1\} \rightarrow \boldsymbol{Z} / a \boldsymbol{Z}$, respectively, which shows that there are $r-a$ different L-bricks and $a$ different R-bricks.

Definition 3.4. For fixed $r$ and $a$, let $Y(r, a)$ denote the Danilov resolution of the $\frac{1}{r}(1, a, r-a)$ singularity.

The rest of this section is devoted to finding effective toric divisors $X_{i}(r, a), Y_{i}(r, a)$, $Z_{i}(r, a)$ on $Y(r, a)$ for $i=0, \ldots, r-1$. These divisors will yield $\boldsymbol{Q}$-divisors $R_{i}(r, a)$, used in defining the structure of a moduli space on $Y(r, a)$. Note that the addition in the indices of $X_{i}(r, a), Y_{i}(r, a), Z_{i}(r, a)$ is always meant modulo $r$. 
DEFINITION 3.5. For fixed $r$ and $a$, let

$$
\begin{gathered}
Y_{i-a}(r, a)=\sum_{k=0}^{\tau(r, a)(i)} D_{k}(r, a) \text { for } i=0, \ldots, r-1, \\
Z_{i}(r, a)=\sum_{k=\tau(r, a)(i)+1}^{r} D_{k}(r, a) \text { for } i=0, \ldots, r-1,
\end{gathered}
$$

and let $X_{i}$ be the divisor defined by the equations $X_{0}=E_{1}$ and

$$
X_{i}(r, a)+Z_{i+1}(r, a)=Z_{i}(r, a)+X_{i-a}(r, a) \text { for } i=0, \ldots, r-1 .
$$

These conditions ensure that the divisors $X_{i}$ are uniquely determined. Note that by definition

$$
Y_{i-a}(r, a)+Z_{i}(r, a)=\sum_{i=0}^{r} D_{k}(r, a),
$$

in particular $Y_{i-a}(r, a)+Z_{i}(r, a)$ does not depend on $i$. Moreover,

$$
X_{i}(r, a)+Y_{i+1}(r, a)=Y_{i}(r, a)+X_{i+a}(r, a) .
$$

REMARK 3.6. To shorten notation, we write $X_{i}, Y_{i}, Z_{i}$ instead of $X_{i}(r, a), Y_{i}(r, a)$, $Z_{i}(r, a)$ when no confusion can arise.

The divisors $X_{i}, Y_{i}, Z_{i}$ satisfy commutativity relations

$$
\begin{gathered}
X_{i}+Y_{i+1}=Y_{i}+X_{i+a}, \\
X_{i}+Z_{i+1}=Z_{i}+X_{i-a}, \\
Y_{i}+Z_{i+a}=Z_{i}+Y_{i-a} .
\end{gathered}
$$

LEMMA 3.7. For $a=1$, we have $Y_{i}=D_{0}+\cdots+D_{i}$ for $i=0, \ldots, r-1$ and $Z_{0}=D_{r}, Z_{i}=D_{i}+\cdots+D_{r}$ for $i=1, \ldots, r-1$. Moreover, $X_{i}=Y_{i}+E_{1}-D_{0}$ for $i=0, \ldots, r-1$. For $a=r-1$, we have $Y_{0}=D_{0}, Y_{i}=D_{0}+\cdots+D_{r-i}$ for $i=1, \ldots, r-1$ and $Z_{i}=D_{r-i}+\cdots+D_{r}, X_{i}=Z_{i}+E_{1}-D_{r}$ for $i=0, \ldots, r-1$.

ProOF. To see this, it is enough to combine Definitions 3.1 and 3.5.

EXAMPLE 3.8. For $r=5$ and $a=2$, we have $\tau(5,2)=(0,4,1,3,2)$ (cf. Example 3.2), and hence

$$
\begin{array}{ll}
Y_{0}=D_{0}, & Z_{0}=D_{5}, \\
Y_{1}=D_{0}+D_{1}+D_{2}, & Z_{1}=D_{4}+D_{5}, \\
Y_{2}=D_{0}+D_{1}, & Z_{2}=D_{1}+D_{2}+D_{3}+D_{4}+D_{5}, \\
Y_{3}=D_{0}+D_{1}+D_{2}+D_{3}+D_{4}, & Z_{3}=D_{3}+D_{4}+D_{5}, \\
Y_{4}=D_{0}+D_{1}+D_{2}+D_{3}, & Z_{4}=D_{2}+D_{3}+D_{4}+D_{5} .
\end{array}
$$


By solving the linear equations $X_{i}+Z_{i+1}=Z_{i}+X_{i-2}$ in $X_{i}$ 's, we have

$$
\begin{aligned}
& X_{0}=E_{1}, \\
& X_{1}=E_{1}+D_{2}+D_{4}, \\
& X_{2}=E_{1}+D_{1}+D_{2}, \\
& X_{3}=E_{1}+D_{4}, \\
& X_{4}=E_{1}+D_{1}+2 D_{2}+D_{2}+D_{3}+D_{4} .
\end{aligned}
$$

Definition 3.9. For fixed $r$, $a$, let $r_{L}=r-a$ and $a_{L}=\langle r\rangle_{r-a}$. By $X_{i}^{L}(r, a)$, $Y_{i}^{L}(r, a), Z_{i}^{L}(r, a)$ we mean the pullbacks of the divisors $X_{i}\left(r_{L}, a_{L}\right), Y_{i}\left(r_{L}, a_{L}\right), Z_{i}\left(r_{L}, a_{L}\right)$ by the rational inverse of the inclusion $Y\left(r_{L}, a_{L}\right) \hookrightarrow Y(r, a)$, respectively. Similarly, let $r_{R}=a$ and $a_{R}=\langle-r\rangle_{a}$. By $X_{i}^{R}(r, a), Y_{i}^{R}(r, a), Z_{i}^{R}(r, a)$ we mean the pullbacks of the divisors $X_{i}\left(r_{R}, a_{R}\right), Y_{i}\left(r_{R}, a_{R}\right), Z_{i}\left(r_{R}, a_{R}\right)$ by the rational inverse of the inclusion $Y\left(r_{R}, a_{R}\right) \hookrightarrow$ $Y(r, a)$, respectively. In particular,

$$
\begin{gathered}
Z_{i}^{L}(r, a)=\sum_{k=\tau\left(r_{L}, a_{L}\right)(i)+1}^{r_{L}} D_{k}(r, a) \text { for } i=0, \ldots, r_{L}-1, \\
Z_{i}^{R}(r, a)=\sum_{k=\tau\left(r_{R}, a_{R}\right)(i)+1}^{r_{R}} D_{k+r_{L}}(r, a) \text { for } i=0, \ldots, r_{R}-1 .
\end{gathered}
$$

When no confusion can arise, we write these divisors by $X_{i}^{L}, Y_{i}^{L}, Z_{i}^{L}, X_{i}^{R}, Y_{i}^{R}, Z_{i}^{R}$ for short. We note that in the definition of $Z_{i}^{R}$ there is a shift by $r_{L}$ in the index of $D_{i}$ since the divisor $D_{i+r_{L}}(r, a)$ on the resolution $Y(r, a)$ corresponds to the divisor $D_{i}\left(r_{R}, a_{R}\right)$ on the resolution $Y\left(r_{R}, a_{R}\right)$ for $i=0, \ldots, r_{R}-1$.

EXAMPLE 3.10. For $r=5$ and $a=2$, by Lemma 3.7, we have

$$
\begin{array}{ll}
Z_{0}^{L}=D_{3}, & X_{0}^{L}=E_{1}, \\
Z_{1}^{L}=D_{2}+D_{3}, & X_{1}^{L}=E_{1}+D_{2}, \\
Z_{2}^{L}=D_{1}+D_{2}+D_{3}, & X_{2}^{L}=E_{1}+D_{1}+D_{2}, \\
Z_{0}^{R}=D_{5}, & X_{0}^{R}=E_{1}, \\
Z_{1}^{R}=D_{4}+D_{5}, & X_{1}^{R}=E_{1}+D_{4} .
\end{array}
$$

Note the indices in the divisors $D_{i}$ 's on the R-resolution are shifted by $r_{L}=3$. Since the L-resolution is a resolution of the $\frac{1}{3}(1,2,1)$ singularity, we have by Lemma 3.7

$$
Y_{0}^{L}=D_{0}, \quad Y_{1}^{L}=D_{0}+D_{1}, \quad Y_{2}^{L}=D_{0}+D_{1}+D_{2} .
$$

On the other hand, the R-resolution is a resolution of the $\frac{1}{2}(1,1,1)$ singularity, hence

$$
Y_{0}^{R}=D_{3}, \quad Y_{1}^{R}=D_{3}+D_{4} .
$$

Following lemmas are useful in later sections. 
Lemma 3.11. Let $i, \ldots, i+s(r-a)$ be an L-brick. The restriction of the divisor $Z_{i+s(r-a)}(r, a)$ to the L-resolution is equal to the divisor $Z_{i}^{L}(r, a)$. If $i, \ldots, i+s a$ is an $R$ brick, then the restriction of the divisor $Z_{i}(r, a)$ to the $R$-resolution is equal to the divisor $Z_{i}^{R}(r, a)$.

Proof. Observe that if $i, \ldots, i+s(r-a)$ is an L-brick, then $i+s(r-a) \geq a$ and $\langle i+s(r-a)\rangle_{r-a}=i$. Therefore $\tau(r, a)(i+s(r-a))=\tau(r-a,\langle r\rangle)(i)$. If $i, \ldots, i+s a$ is an R-brick, then $i<a$ and $\tau(r, a)(i)=(r-a)+\tau(a,\langle-r\rangle)(i)$.

Similar facts hold for the restriction of the divisors $X_{i}$.

Lemma 3.12. For any $i \leq r-2$, the divisor $X_{i}(r, a)$ restricted to the L-resolution is equal to the divisor $X_{j}^{L}(r, a)$ for $j=\langle i\rangle_{r-a}$, and the divisor $X_{i}(r, a)$ restricted to the $R$-resolution is equal to the divisor $X_{j}^{R}(r, a)$ for $j=\langle i\rangle_{a}$.

Proof. We give the proof only in the case of the restriction to the R-resolution. First we show that if $i, \ldots, i+s a$ is an R-brick such that $i+s a \neq r-1$, then the R-restrictions of the divisors $X_{i}, \ldots, X_{i+s a}$ are equal to each other. To see this, observe that the restrictions of the divisors $Z_{j}$ for $j \geq a$ to the R-resolution are equal to each other (cf. Definitions 3.1 and 3.5). This, combined with the commutativity relations (3.2),

$$
Z_{j}-Z_{j+1}=X_{j}-X_{j-a}
$$

for $j=i+a, i+2 a, \ldots, i+s a$, implies that the R-restrictions of the divisors $X_{i}, \ldots, X_{i+s a}$ are equal to each other.

Similarly, if $i, \ldots, i+s a$ is an R-brick such that $i+s a=r-1$, then the restrictions of the divisors $X_{i}, \ldots, X_{i+(s-1) a}$ (i.e., all but the last) to the R-resolution are equal to each other.

Hence, it is enough to prove the lemma assuming $i<a$. Denote by $\left.X_{i}\right|_{R}$ the restriction of the divisor $X_{i}$ to the R-resolution. Obviously $\left.X_{0}\right|_{R}=X_{0}^{R}$ and by Lemma 3.11 we obtain relations

$$
\left.X_{i}\right|_{R}+Z_{i+1}^{R}=Z_{i}^{R}+\left.X_{i-a}\right|_{R}
$$

for $i=0, \ldots, a-2$. We have proven already that $\left.X_{i-a}\right|_{R}=\left.X_{\langle i+r\rangle_{a}}\right|_{R}$, and so the above relations can be rewritten as

$$
\left.X_{i}\right|_{R}+Z_{i+1}^{R}=Z_{i}^{R}+\left.X_{\langle i+r\rangle_{a}}\right|_{R} .
$$

These are exactly the equations (3.2) for $r_{R}=a$ and $a_{R}=\langle-r\rangle_{a}$, and we have

$$
\left.X_{i}\right|_{R}=X_{i}^{R} \quad \text { for } \quad i=0, \ldots, a-2 .
$$

Let $j$ be the last element of an R-brick containing $a-1$. Then $j \neq r-1$ so $0 \leq$ $\langle j+a\rangle_{r}<a-1$, and the equation (3.2) for $i=j+a$

$$
X_{j+a}+Z_{j+a+1}=X_{j}+Z_{j+a},
$$

restricted to the R-resolution becomes

$$
X_{j+a-r}^{R}+Z_{j+a-r+1}^{R}=\left.X_{a-1}\right|_{R}+Z_{j+a-r}^{R} .
$$


This finishes the proof since the above is exactly the equation (3.2) for $r_{R}=a, a_{R}=\langle-r\rangle_{a}$ and $i=j+a-r$.

EXAMPLE 3.13. For $r=5$ and $a=2$, there are 3 different L-bricks $(0,3),(1,4),(2)$ and 2 different R-bricks $(0,2,4),(1,3)$. The L-resolution contains divisors $D_{0}, D_{1}, D_{2}, D_{3}$, $E_{1}$ and the R-resolution contains divisors $D_{3}, D_{4}, D_{5}, E_{1}$. By Examples 3.8 and 3.10, we note that

$$
\begin{gathered}
\left.Z_{3}\right|_{L}=Z_{0}^{L},\left.\quad Z_{4}\right|_{L}=Z_{1}^{L},\left.\quad Z_{2}\right|_{L}=Z_{2}^{L}, \\
\left.Z_{0}\right|_{R}=Z_{0}^{R},\left.\quad Z_{1}\right|_{R}=Z_{1}^{R} .
\end{gathered}
$$

Moreover,

$$
\begin{gathered}
\left.X_{0}\right|_{L}=X_{3}^{L}=X_{0}^{L},\left.\quad X_{0}\right|_{R}=\left.X_{2}\right|_{R}=X_{0}^{R}, \\
\left.X_{1}\right|_{L}=X_{1}^{L},\left.\quad X_{1}\right|_{R}=X_{1}^{R} .
\end{gathered}
$$

LEMMA 3.14. For a, $r$ coprime, the divisors $X_{i}-E_{1}, Y_{i}-E_{2}, Z_{i}-E_{3}$ are effective for $i=0, \ldots, r-1$.

PRoOF. The result is true for $Y_{i}-E_{2}$ and $Z_{i}-E_{3}$ by definition, since $E_{2}=D_{0}$ and $E_{3}=D_{r}$. It remains to prove the result for $X_{i}-E_{1}$.

Note that for $a \in\{1, r-1\}$ either $X_{i}-E_{1}=Y_{i}-E_{2}$ or $X_{i}-E_{1}=Z_{i}-E_{3}$ by Lemma 3.7. For $1<a<r-1$, by recursion and Lemma 3.12, the restrictions of $X_{i}-E_{1}$ to $\mathrm{L}-$ and R-resolution are effective for $i \neq r-1$. Finally, note that

$$
X_{r-1}-E_{1}=\left(X_{r-a-1}-E_{1}\right)+\left(Z_{r-1}-Z_{0}\right),
$$

where both summands are effective, since $Z_{0}=D_{r}$ for any $r, a$.

DEFINITION 3.15. For fixed $r$ and $a$, let

$$
D_{X}(r, a)=\frac{1}{r} \operatorname{div}\left(r e_{1}^{*}\right), \quad D_{Y}(r, a)=\frac{1}{r} \operatorname{div}\left(r e_{2}^{*}\right), \quad D_{Z}(r, a)=\frac{1}{r} \operatorname{div}\left(r e_{3}^{*}\right)
$$

be $Q$-divisors on $Y(r, a)$ where $\operatorname{div}\left(r e_{i}^{*}\right)$ denotes the divisor of zeros and poles of the rational function $r e_{i}^{*}$. We write them by $D_{X}, D_{Y}, D_{Z}$ for short.

We introduce the $Q$-divisors $R_{i}(r, a)$ which later will define the desired family of McKay quiver representations on $Y(r, a)$.

Definition 3.16. For fixed $r$ and $a$, define the $Q$-divisors $R_{i}(r, a)$ for $i=0, \ldots, r-$ 1 by the equations

$$
Z_{i}(r, a)=D_{Z}(r, a)+R_{i}(r, a)-R_{i-a}(r, a), \quad R_{0}(r, a)=0 .
$$

We write $R_{i}$ for short when no confusion can arise.

The divisors $R_{i}$ are uniquely determined by the condition $R_{0}=0$ since $r, a$ are coprime and the rank of the matrix determining equations for $R_{i}$ is equal to $r-1$. Using the equation $Z_{i}+Y_{i-a}=D_{Y}+D_{Z}$ we get $Y_{i}=D_{Y}+R_{i}-R_{i+a}$. 
LEMMA 3.17. For any coprime $r$ and $a$, we have

$$
R_{1}=D_{X}-E_{1} \text {. }
$$

Proof. By definition, we have

$$
\begin{aligned}
R_{1} & =(r-b) D_{Z}-\left(Z_{0}+Z_{-a}+Z_{-2 a}+\cdots+Z_{-(r-b-1) a}\right) \\
& =(r-b) D_{Z}-\left(Z_{a+1}+Z_{2 a+1}+Z_{3 a+1}+\cdots+Z_{0}\right)
\end{aligned}
$$

since $\langle-(r-b-i) a\rangle_{r}=i a+1$. Therefore it is enough to show that

$$
E_{1}=D_{X}-(r-b) D_{Z}-\left(Z_{a+1}+Z_{2 a+1}+\cdots+Z_{0}\right) .
$$

Assume that $a \notin\{1, r-1\}$ as otherwise the statement is trivial (cf. Lemma 3.7). We use a recursive argument. Observe that the numbers in the sequence

$$
\langle a+1\rangle_{r},\langle 2 a+1\rangle_{r},\langle 3 a+1\rangle_{r}, \ldots,\langle 0\rangle_{r}
$$

not greater than $a-1$ (i.e., the first numbers in R-bricks) are equal to the numbers

$$
\left\langle a_{R}+1\right\rangle_{a},\left\langle 2 a_{R}+1\right\rangle_{a},\left\langle 3 a_{R}+1\right\rangle_{a}, \ldots,\langle 0\rangle_{a},
$$

where $a_{R}=\langle-r\rangle_{a}$. Moreover, the numbers in the sequence ( $\star$ ) greater or equal to $a$ (i.e., the last numbers in L-bricks) are equal modulo $r-a$ to the numbers

$$
\left\langle a_{L}+1\right\rangle_{r-a},\left\langle 2 a_{L}+1\right\rangle_{r-a},\left\langle 3 a_{L}+1\right\rangle_{r-a}, \ldots,\langle 0\rangle_{r-a},
$$

where $a_{L}=\langle r\rangle_{r-a}$. We omit a proof of this arithmetic fact. Let $W^{L}, W^{R}$ denote the pullbacks of the divisors $D_{X}\left(r_{L}, a_{L}\right)-\left(r_{L}-b_{L}\right) D_{Z}\left(r_{L}, a_{L}\right)$ and $D_{X}\left(r_{R}, a_{R}\right)-\left(r_{R}-b_{R}\right) D_{Z}\left(r_{R}, a_{R}\right)$ (where $a_{L} b_{L}=1$ modulo $r_{L}$ and $a_{R} b_{R}=1$ modulo $r_{R}$ ) by the rational inverses of the inclusions $Y\left(r_{L}, a_{L}\right) \hookrightarrow Y(r, a)$ and $Y\left(r_{R}, a_{R}\right) \hookrightarrow Y(r, a)$, respectively. Note that the first coordinate of the point $p_{i+1}$ is not smaller than the first coordinate of the point $p_{i}$ if and only if the toric ray dual to the cone $\left\langle p_{i}, p_{i+1}\right\rangle$ is equal to $e_{1}^{*}-(r-b) e_{3}^{*}$, which gives an intrinsic explanation of the value of $e_{1}^{*}-(r-b) e_{3}^{*}$ on the generator of a ray. Since $\left(e_{1}^{*}-(r-\right.$ b) $\left.e_{3}^{*}\right)\left(\frac{1}{r}(1, a, r-a)\right)=b_{L}-r_{L}$, we get $D_{X}-(r-b) D_{Z}=W^{L}+\left(b_{L}-r_{L}\right) W^{R}-E_{1}$, as $E_{1}$ is counted twice. To finish the proof, observe that in the sequence ( $\star$ ) exactly $r_{L}-b_{L}$ numbers are greater than or equal to $a$ and use Lemma 3.11.

LEMMA 3.18. For fixed $r$ and $a$, the divisors $R_{i}$ satisfy the following equations for $i=0, \ldots, r-1$.

$$
\begin{aligned}
& X_{i}=D_{X}+R_{i}-R_{i+1}, \\
& Y_{i}=D_{Y}+R_{i}-R_{i+a}, \\
& Z_{i}=D_{Z}+R_{i}-R_{i-a} .
\end{aligned}
$$

PROOF. It is enough to prove the first equality. Set $\widetilde{X}_{i}=D_{X}+R_{i}-R_{i+1}$ and note that $\sum \widetilde{X_{i}}=r D_{X}$. Moreover, the divisors $\widetilde{X}_{i}$ satisfy the commutativity relations (3.2), and hence $X_{i}-\widetilde{X_{i}}$ is constant. Since $\widetilde{X_{0}}=D_{X}-R_{1}=X_{0}$ by Lemma 3.17, the constant is equal to 0 . 
4. The McKay quiver. By a quiver we mean a finite directed graph $Q$. The set of vertices of $Q$ will be denoted by $Q_{0}$ and the set of arrows by $Q_{1}$. For any arrow $a$ in $Q_{1}$ denote by $\mathrm{t}(a)$ the tail of $a$ and by h $(a)$ the head of $a$. In what follows, we restrict the general definition of quiver representations to the simple case where the dimension vector is equal to $(1, \ldots, 1)$. For any $v \in Q_{0}$, let $\boldsymbol{C}_{v}$ denote 1 -dimensional complex vector space assigned to the vertex $v$. A representation of the quiver $Q$ is an element of

$$
\operatorname{Rep}(Q)=\bigoplus_{a \in Q_{1}} \operatorname{Hom}_{C}\left(\boldsymbol{C}_{\mathrm{t}(a)}, \boldsymbol{C}_{\mathrm{h}(a)}\right) .
$$

By fixing a basis in each $\boldsymbol{C}_{v}$ we can identify $\operatorname{Rep}(Q)$ with an affine space. With this choice, for any representation $V \in \operatorname{Rep}(Q)$ and $a \in Q_{1}$ denote by $V(a)$ the constant representing the linear map for the arrow $a$ in $V$.

A path $q$ in quiver $Q$ is a sequence of arrows $a_{l}, \ldots, a_{2}, a_{1}$ where $\mathrm{h}\left(a_{i}\right)=\mathrm{t}\left(a_{i+1}\right)$. For such path $q$ and for any representation $V$ of $Q$ define $V(q)=V\left(a_{l}\right) \cdots V\left(a_{1}\right)$. A linear combination of paths $q_{i}$ is called an admissible relation, if paths $q_{i}$ have the same heads and tails. Any set $R$ of admissible relations for a quiver $Q$ defines an affine subscheme of $\operatorname{Rep}(Q)$ cut by the polynomial equations coming from $R$, i.e.,

$$
\operatorname{Rep}(Q, R):=\{V \in \operatorname{Rep}(Q) ; V(c)=0 \quad \text { for } c \in R\},
$$

where the function $V(c)$ denotes the linear extension of the functions $V\left(q_{i}\right)$.

Two representations of a quiver $Q$ are isomorphic if and only if they lie in the same orbit of the group $\operatorname{GL}(Q, \boldsymbol{C})=\bigoplus_{v \in Q_{0}} \boldsymbol{C}^{*}$, acting from the left on the set $\operatorname{Rep}(Q)$ in the way

$$
(g \cdot V)(a)=g(\mathrm{~h}(a)) V(a) g(\mathrm{t}(a))^{-1} \quad \text { for any } \quad V \in \operatorname{Rep}(Q) .
$$

This action leaves $\operatorname{Rep}(Q, R)$ invariant. Dividing by the 1-dimensional subgroup acting trivially, we are left with a faithful action of the group

$$
\operatorname{PGL}(Q, C)=\operatorname{GL}(Q, C) / \boldsymbol{C}^{*}(1, \ldots, 1) .
$$

Definition 4.1. By a subquiver $Q^{\prime} \subset Q$ we mean a subset of vertices $Q_{0}^{\prime} \subset Q_{0}$ and a subset of arrows $Q_{1}^{\prime} \subset Q_{1}$ satisfying $\mathrm{t}(a), \mathrm{h}(a) \in Q_{0}^{\prime}$ for any $a \in Q_{1}^{\prime}$. Let $V \in \operatorname{Rep}(Q, R)$ be a representation of $Q$. A subrepresentation $V^{\prime}$ of $V$ is a representation of a subquiver $Q^{\prime} \subset Q$ satisfying

$$
V^{\prime}(a)=V(a) \quad \text { for any } a \in Q_{1}^{\prime} .
$$

We do not need the general definition of the McKay quiver, so we quote only the specialization to the case of a cyclic group action.

Definition 4.2. (McKay) Let $G$ be a cyclic group $G \subset \mathrm{GL}(3, \boldsymbol{C})$ of order $r$, such that the quotient singularity $C^{3} / G$ is of type $\frac{1}{r}(1, a, r-a)$. Define the McKay quiver for $G$ as a finite graph with $r$ vertices $0,1, \ldots, r-1$ and $3 r$ arrows $x_{0}, y_{0}, z_{0}, \ldots, x_{r-1}, y_{r-1}, z_{r-1}$ such that $\mathrm{t}\left(x_{i}\right)=\mathrm{t}\left(y_{i}\right)=\mathrm{t}\left(z_{i}\right)=i$ and

$$
\mathrm{h}\left(x_{i}\right)=\langle i+1\rangle_{r}, \quad \mathrm{~h}\left(y_{i}\right)=\langle i+a\rangle_{r}, \quad \mathrm{~h}\left(z_{i}\right)=\langle i-a\rangle_{r} .
$$

The vertices of the McKay quiver correspond to the characters of $G$. 


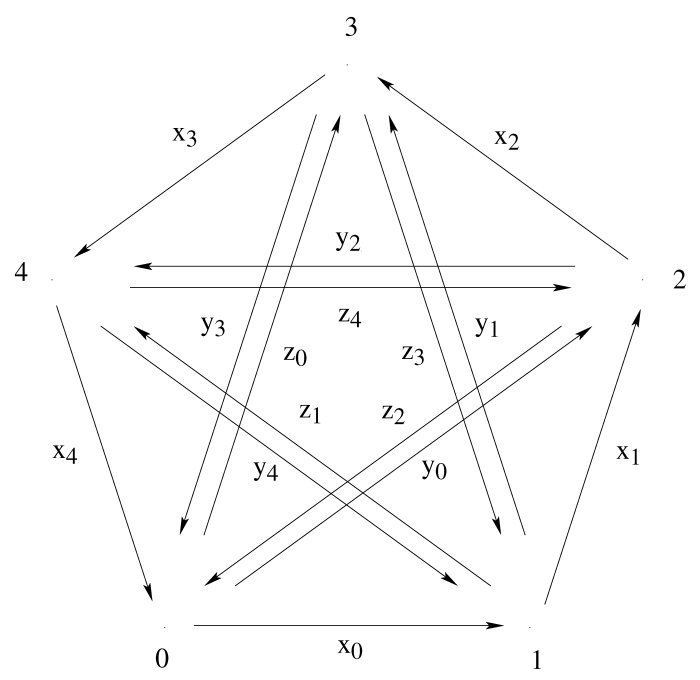

FIGURE 2. The McKay quiver for $r=5, a=2$.

Definition 4.3. A representation of the McKay quiver is an element of $\operatorname{Rep}(Q, R)$, where $Q$ is the McKay quiver (for fixed $r, a$ ) and $R$ is the set relations

$$
R=\left\{y_{i+1} x_{i}-x_{i+a} y_{i}, z_{i+1} x_{i}-x_{i-a} z_{i}, y_{i-a} z_{i}-z_{i+a} y_{i} ; i=0, \ldots, r-1\right\},
$$

where all indices are meant modulo $r$.

5. Family of representations of the McKay quiver. In this section we will define a family of the McKay quiver representations over the Danilov resolution using line bundles determined by the effective divisors $X_{i}, Y_{i}, Z_{i}$.

Definition 5.1 (King, Logvinenko). Fix coprime $r, a$ and let $Y(r, a)$ be the Danilov resolution. By a family of McKay quiver representations on $Y(r, a)$ for the action of type $\frac{1}{r}(1, a, r-a)$, we mean a set of $r \boldsymbol{Q}$-divisors $R_{i}$ for $i=0, \ldots, r-1$ on $Y(r, a)$, such that the $\boldsymbol{Q}$-divisors

$$
X_{i}=D_{X}+R_{i}-R_{i+1}, \quad Y_{i}=D_{Y}+R_{i}-R_{i+a}, \quad Z_{i}=D_{Z}+R_{i}-R_{i-a}
$$

are effective divisors for $i=0, \ldots, r-1$ (where $D_{X}, D_{Y}, D_{Z}$ are as in Definition 3.15).

The above condition is called the reductor condition in [13]. It ensures that the family of quiver representations on the resolution $Y(r, a)$ is natural, that is, the support of a representation (seen as a finite dimensional $\boldsymbol{C}[x, y, z]$-module) parameterized by a point $p$ in the resolution coincides with the $G$-orbit parameterized by the image of $p$ in the quotient space (cf. [13, Definition 1.4]).

Definition 5.2. For fixed $a$ and $r$, denote by $\mathcal{F}(r, a)$ (or $\mathcal{F}$ for short) the family given by the $Q$-divisors $R_{i}(r, a)$ in Definition 3.16. 
REMARK 5.3. Note that the divisors $R_{i}$ satisfy the reductor condition by Lemma 3.18 and the divisors $X_{i}, Y_{i}, Z_{i}$ are effective by Lemma 3.14.

One could define the family $\mathcal{F}(r, a)$ by representing the arrows of the McKay quiver, on each affine toric chart, by the corresponding character of the piecewise linear function given by the effective Cartier divisors $X_{i}, Y_{i}$ and $Z_{i}$ from Definition 5.1. Note that in this case every such character is a regular function on the toric chart. Equivalently, define line bundles $L_{i}=\mathcal{O}_{Y}\left(-R_{i}\right)$ on $Y$ for $0 \leq i \leq r-1$. Then, for each $0 \leq i \leq r-1$, the multiplications by the sections defining the divisors $X_{i}, Y_{i}$ and $Z_{i}$, respectively, determine morphisms from $L_{i}$ to $L_{i+1}$, to $L_{i+a}$ and to $L_{i-a}$, respectively.

We will show later that there exist stability conditions $\theta$ such that every representation in the family $\mathcal{F}(r, a)$ is $\theta$-(semi)stable. In fact, it will turn out that such stability conditions $\theta$ are exactly those for which the representations parameterized by $T$-fixed point of the cones $\sigma_{0}, \ldots, \sigma_{r-1}$ are simultaneously $\theta$-(semi)stable.

Definition 5.4. For the family $\mathcal{F}$ from Definition 5.2 and for any 3-dimensional cone $\sigma$ in the fan of the Danilov resolution, we call an arrow in the McKay quiver $\sigma$ distinguished if the corresponding divisor (that is, $X_{i}, Y_{i}$ or $Z_{i}$ ) does not contain the $T$-fixed point of toric chart $U_{\sigma}$.

Observe, for example, that no $z_{i}$-arrow is $\sigma_{r-1}$-distinguished since the divisors $Z_{i}-E_{3}$ are effective for any $i$.

LEMMA 5.5. For any 3-dimensional cone $\sigma$ in the fan of the Danilov resolution, any two vertices of the McKay quiver can be connected by an undirected path of $\sigma$-distinguished arrows different from $x_{r-1}$.

Proof. The lemma is true for $a \in\{1, r-1\}$. Note that any two vertices of the McKay quiver lying in the same L-brick can be joined by a sequence of $z$-arrows for any L-cone $\sigma$, and any two vertices lying in the same R-brick can be joined by a sequence of $y$-arrows if $\sigma$ is an $\mathrm{R}$-cone. By the inductive step, any two bricks can be joined by a sequence of $\sigma$-distinguished arrows. It is enough to consider the cone $\sigma=\left\langle p_{0}, p_{r-a}, p_{r}\right\rangle$, and we are done since the only $\sigma$-distinguished arrows are $x_{0}, \ldots, x_{r-2}$.

Lemma 5.6. Let $p, p^{\prime} \in Y$ be two points in the Danilov resolution, belonging to two distinct toric charts isomorphic to $\boldsymbol{C}^{3}$. Then the representations parameterized by $p$ and $p^{\prime}$ in $\mathcal{F}$ are not isomorphic.

Proof. Let $\sigma$ and $\sigma^{\prime}$ be 3-dimensional cones in the fan of the Danilov resolution corresponding to charts containing $p$ and $p^{\prime}$, respectively. There are at most two common primitive generators of the cones $\sigma$ and $\sigma^{\prime}$ belonging to the set $\left\{p_{0}, \ldots, p_{r}\right\}$. This implies that at least one $y$ - or $z$-arrow is $\sigma$-distinguished and not $\sigma^{\prime}$-distinguished. Hence the representations parameterized by $p$ and $p^{\prime}$ in $\mathcal{F}$ are not isomorphic. 
Lemma 5.7. Let $p, p^{\prime} \in Y$ be distinct points in the Danilov resolution belonging to a single toric chart, isomorphic to $\boldsymbol{C}^{3}$, on the Danilov resolution. The representations parameterized by $p$ and $p^{\prime}$ in $\mathcal{F}$ are not isomorphic.

Proof. Let $\sigma=\left\langle p_{l}, p_{m}, p_{n}\right\rangle$ be the 3-dimensional cone in the fan of Danilov resolution such that $p, p^{\prime} \in U_{\sigma}$, where $U_{\sigma}$ stands for the toric chart given by $\sigma$. We claim that there exist $i, j, k \in\{0, \ldots, r-1\}, i \neq r-1$, such that the restrictions of $X_{i}, Y_{j}, Z_{k}$ to the chart $U_{\sigma}$ are equal to the restrictions of the divisors $\left.D_{l}\right|_{U_{\sigma}},\left.D_{m}\right|_{U_{\sigma}},\left.D_{n}\right|_{U_{\sigma}}$, respectively. This holds for $a \in\{1, r-1\}$ and can be proven for $a \notin\{1, r-1\}$ using recursion and Lemmas 3.11 and 3.12. In the orbit of the group $\operatorname{GL}(Q, \boldsymbol{C})$, there exists exactly one representation such that all $\sigma$-distinguished arrows are represented by the number 1 (by Lemma 5.5). Therefore, in this unique element of the orbit, the arrows $x_{i}, y_{j}, z_{k}$ are represented by toric coordinates on $U_{\sigma}$. The points $p, p^{\prime}$ have at least one different toric coordinate. Therefore they parameterize non-isomorphic representations.

COROLlary 5.8. For any two distinct points $p, p^{\prime} \in Y$ in the Danilov resolution, the representations parameterized by $p$ and $p^{\prime}$ in $\mathcal{F}$ are not isomorphic.

Definition 5.9. For fixed coprime $r$ and $a$, let $\mathcal{F}_{i}$ denote the representation of the McKay quiver parameterized in the family $\mathcal{F}(r, a)$ by the unique $T$-fixed point belonging to the toric chart $U_{\sigma_{i}}$ for $i=0, \ldots, r-1$ (cf. Definition 2.5).

Since the divisors $X_{j}-E_{1}$ are effective, no $x_{j}$-arrow is $\sigma_{i}$-distinguished for any $i$. Moreover, by the definition of the permutation $\tau$, for any $i$ there exists a unique $j$ such that $y$-arrow and $z$-arrow joining vertices $j$ and $j+a$ are not $\sigma_{i}$-distinguished. For $i$ and $j$ as above, if $j^{\prime} \neq j$, then among the $y$ - and $z$-arrows joining $j^{\prime}$ and $j^{\prime}+a$ exactly once is $\sigma_{i}$-distinguished (cf. Example 7.4). Hence the representations $\mathcal{F}_{i}$ are particularly easy to deal with.

6. Stability of quiver representations. In this section, we recall some facts and definitions concerning $\theta$-stability of quiver representations (see [12]), note that we restrict to the case of dimension vector equal to $(1, \ldots, 1)$. We prove that the representations in the family $\mathcal{F}$ (cf. Definition 5.2) on the Danilov resolution are simultaneously $\theta$-(semi)stable if and only if the representation $\mathcal{F}_{0}, \ldots, \mathcal{F}_{r-1}$ are $\theta$-(semi)stable.

For any quiver $Q$, set

$$
\mathrm{Wt}(Q)=\left\{\theta: Q_{0} \rightarrow \boldsymbol{Q} ; \sum_{v \in Q_{0}} \theta(v)=0\right\} .
$$

Given a function $\theta \in \mathrm{Wt}(Q)$ for which $\theta\left(Q_{0}\right) \subset \boldsymbol{Z}$, we obtain a character $\chi_{\theta}$ of $\operatorname{PGL}(Q, \boldsymbol{C})$. Explicitly, for any such $\theta$, the character is

$$
\chi_{\theta}(g)=\prod_{v \in Q_{0}} g(v)^{\theta(v)},
$$

where $g \in \operatorname{PGL}(Q, \boldsymbol{C})$. Therefore, we will call $\mathrm{Wt}(Q)$ the weight space for $Q$. 
Definition 6.1. (A. King) For any subrepresentation $V^{\prime}$ of a representation $V \in$ $\operatorname{Rep}(Q)$ and $\theta \in \operatorname{Wt}(Q)$, set

$$
\theta\left(V^{\prime}\right)=\sum_{v \in Q_{0}^{\prime}} \theta(v)
$$

A representation $V$ is called $\theta$-semistable if $\theta\left(V^{\prime}\right) \geq \theta(V)=0$ for every proper non-zero subrepresentation $V^{\prime} \subset V$. A representation $V$ is called $\theta$-stable if an analogous condition with strict inequality holds.

Definition 6.2. We say that a stability parameter $\theta \in \mathrm{Wt}(Q)$ is generic if every $\theta$-semistable representation is $\theta$-stable.

THEOREM 6.3 (King). Let $Q$ be a quiver, $R$ a set of admissible relations for $Q$ and let $\theta \in \mathrm{Wt}(Q)$. A point in $\operatorname{Rep}(Q, R)$ is $\chi_{\theta}$-(semi)stable under the action of $\operatorname{PGL}(Q, C)$ if and only if the corresponding representation of $Q$ is $\theta$-(semi)stable. Denote by $M_{\theta}(Q, R)$ the GIT quotient of $\operatorname{Rep}(Q, R)$ by $\operatorname{PGL}(Q, C)$ with respect to the $\chi_{\theta}$-linearization of the trivial bundle over $\operatorname{Rep}(Q, R)$. That is, $M_{\theta}(Q, R)$ is the scheme constructed from the graded ring of semi-invariants, namely,

$$
M_{\theta}(Q, R):=\operatorname{Rep}(Q, R) / /_{\chi_{\theta}} \operatorname{PGL}(Q, C)=\operatorname{Proj} \bigoplus_{k=0}^{\infty} \boldsymbol{C}[\operatorname{Rep}(Q, R)]^{\chi_{\theta}^{k}},
$$

where elements of the set $C[\operatorname{Rep}(Q, R)]^{\chi}$ are regular functions $f$ on the representation space $\operatorname{Rep}(Q, R)$, such that $f(g \cdot v)=\chi(g) f(v)$ for any $g \in \operatorname{PGL}(Q, C)$ and any $v \in \operatorname{Rep}(Q, R)$.

For a generic $\theta$, the scheme $M_{\theta}(Q, R)$ is a fine moduli space of $\theta$-stable representations.

ProOF. See [12, Propositions 3.1, 5.2, 5.3].

We need a fact concerning families of $\theta$-(semi)stable quiver representations on affine toric varieties, which is true in a more general setting.

LEMMA 6.4. Let $U_{\sigma}$ be an affine toric chart in the Danilov resolution $Y$ containing a unique $T$-fixed point $p_{\sigma}$. Let $\mathcal{F}$ be a family of McKay quiver representations on $Y$ as in Definition 5.1. If the representation parameterized by the point $p_{\sigma}$ is $\theta$-(semi)stable, then all representation in $\mathcal{F}$ parameterized by closed points in $U_{\sigma}$ are $\theta$-(semi) stable.

ProOf. The $\theta$-(semi)stability is an open condition and it is invariant under the $T$-action since the divisors $R_{i}$ are $T$-equivariant. Moreover, the $T$-fixed point lies in the closure of all orbits in $U_{\sigma}$.

We need also two simple lemmas.

LEMMA 6.5. Let $U_{\sigma}$ be a toric chart in the Danilov resolution, where $\sigma=\left\langle p_{l}, p_{m}, p_{n}\right\rangle$ and $l<m<n$. If the arrow $y_{i}$ is $\sigma$-distinguished, then it is $\sigma_{j}$-distinguished for $j \geq l$. If the arrow $y_{i}$ is not $\sigma$-distinguished, then it is not $\sigma_{j}$-distinguished for $j \leq l$. If the arrow $z_{i}$ is $\sigma$-distinguished, then it is $\sigma_{j}$-distinguished for $j<n$. If the arrow $z_{i}$ is not $\sigma$-distinguished, then it is not $\sigma_{j}$-distinguished for $j \geq n-1$. 
PROOF. It follows directly from Definition 3.5.

LEMMA 6.6. Let $i, i+a, \ldots, i+$ sa be an $R$-brick. Then, for $j=i, i+(r-a), \ldots, i+$ $(s-1)(r-a)$, the $y_{j}$-arrows are $\sigma$-distinguished for any cone $\sigma$ in the R-resolution. Let $i, i+(r-a), \ldots, i+s(r-a)$ be an L-brick. Then, for $j=i+a, \ldots, i+s(r-a)$, the $z_{j}$-arrows are $\sigma$-distinguished for any cone $\sigma$ in the $L$-resolution.

Proof. Let $i, i+(r-a), \ldots, i+s(r-a)$ be an L-brick. Then, $i+k(r-a)<a$ for $k=0, \ldots, s-1$. Hence, by Definition 3.1, $\tau(r, a)(i+k(r-a)) \geq r-a$. This implies that the supports of divisors $Z_{i}, Z_{i+(r-a)}, \ldots, Z_{i+s(r-a)}$ are disjoint from any torus-fixed point on the L-resolution. The other case follows analogously.

Using the above tools, we can prove that $\theta$-stability of the representations $\mathcal{F}_{1}, \ldots, \mathcal{F}_{r-1}$ (see Definition 5.9) controls stability of the whole family $\mathcal{F}$.

LEMMA 6.7. Let $\theta$ be a stability parameter such that $\mathcal{F}_{0}, \ldots, \mathcal{F}_{r-1}$ are $\theta$-(semi) stable. Then every representation in the family $\mathcal{F}$ is $\theta$-(semi)stable.

PROOF. By Lemma 6.4, to conclude, it is enough to prove that every representation in $\mathcal{F}$, parameterized by a $T$-fixed point, is $\theta$-(semi)stable.

Let $p_{\sigma} \in U_{\sigma}$ be a $T$-fixed point, where $\sigma=\left\langle p_{l}, p_{m}, p_{n}\right\rangle$ is a 3-dimensional cone in the fan of the Danilov resolution and $l<m<n$. Let $V$ be a subrepresentation of the representation parameterized by $p_{\sigma}$ in the family $\mathcal{F}$. The proof is done by induction on $r$. We will show that there exists $j \in\{0, \ldots, r-1\}$ and a subrepresentation of $\mathcal{F}_{j}$ supported on the same set of vertices as $V$.

The theorem is trivial if $a \in\{1, r-a\}$. Assume that $r>1$ and the theorem is true for any $r^{\prime}<r$. Let $V$ be a subrepresentation as above. By $S(V) \subset\{0, \ldots, r-1\}$ we mean a subset of the vertices of the McKay quiver, supporting $V$. Consider a sequence $i, i+(r-a), \ldots, i+$ $s(r-a)$ of vertices in the set $S(V)$, such that the vertices $i-(r-a), i+(s+1)(r-a)$ are not in $S(V)$. The set $S(V)$ is a union of such sequences. There is no loss of generality in

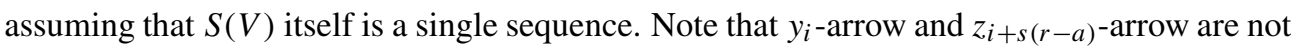
$\sigma$-distinguished ( $V$ is a subrepresentation, see Definition 4.1).

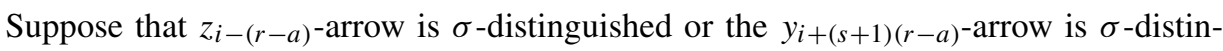
guished. We can assume that $k<r+1$, otherwise there is nothing to prove. By Lemma 6.5, the vertices $i, \ldots, i+s(r-a)$ form a subrepresentation of some of the representations $\mathcal{F}_{i}, \ldots, \mathcal{F}_{k-1}$.

Now we turn to the case when both the $z_{i-(r-a)}$-arrow and the $y_{i+(s+1)(r-a)}$-arrow are not $\sigma$-distinguished. Assume that $\sigma$ is an L-cone. Since the $y_{i}$-arrow and the $z_{i+s(r-a)^{-}}$ arrow are not $\sigma$-distinguished, the sequence $i, \ldots, i+s(r-a)$ is concatenated out of some L-bricks by Lemma 6.6. These L-bricks correspond to vertices of the McKay quiver for $(1 /(r-a))(1,\langle r\rangle,\langle-r\rangle)$. Moreover, the vertices corresponding to these L-bricks form a subrepresentation of the representation parameterized by $p_{\sigma}$ in the family $\mathcal{F}(r-a,\langle r\rangle)$ on the L-resolution. Now we can use the inductive assumption. 
7. Stability of the representations $\mathcal{F}_{0}, \ldots, \mathcal{F}_{r-1}$. We proved that every representation in the family $\mathcal{F}$ is $\theta$-(semi)stable if and only if the representations $\mathcal{F}_{0}, \ldots, \mathcal{F}_{r-1}$ are simultaneously $\theta$-(semi)stable. We will show how to get such parameters $\theta$ using permutation $\tau$.

Definition 7.1. Let $\xi(r, a)=\tau(r, a)^{-1}$ denote the inverse of the permutation $\tau$ (see Definition 3.1).

Since no $x_{i}$-arrow is $\sigma_{j}$-distinguished, any two vertices of $\mathcal{F}_{j}$ can be joined by a sequence of $z$ - and $y$-arrows by Lemma 5.5. Moreover, the arrows $z_{\xi(r, a)(j)}$ and $y_{\xi(r, a)(j)+(r-a)}$ are not $\sigma_{j}$-distinguished. Therefore, the quiver supporting representation $\mathcal{F}_{j}$ consists of vertices $0,1, \ldots, r-1$ and every two vertices $i, i+(r-a)$ are joined either by a $z$-arrow or a $y$-arrow (but not both) unless $i=\xi(r, a)(j)$ (cf. Example 7.4).

DEFINITION 7.2. Let

$\bar{V}_{j}=\left\{i\right.$; vertices $i, i+(r-a), \ldots, \xi(r, a)(j)$ of $Q$ form a subrepresentation of $\left.\mathcal{F}_{j}\right\}$,

$\bar{W}_{j}=\left\{i\right.$; vertices $\xi(r, a)(j)+(r-a), \ldots, i$ of $Q$ form a subrepresentation of $\left.\mathcal{F}_{j}\right\}$.

For any $i \in \bar{V}_{j}$, let $V_{i, j}$ be the subrepresentation of $\mathcal{F}_{j}$ consisting of vertices $i, i+(r-$ $a), \ldots, \xi(r, a)(j)$. For any $i \in \bar{W}_{j}$, let $V_{i, j}$ be the subrepresentation of $\mathcal{F}_{j}$ consisting of vertices $\xi(r, a)(j)+(r-a), \ldots, i-(r-a), i$.

Note that $i \in \bar{V}_{j}$ if and only if the $y_{i}$-arrow is not $\sigma_{j}$-distinguished, and $i \in \bar{W}_{j}$ if and only if the $z_{i}$-arrow is not $\sigma_{j}$-distinguished.

LEMMA 7.3. Let $\theta \in \mathrm{Wt}(Q)$ be a fixed stability parameter. The representation $\mathcal{F}_{j}$ is $\theta$-semistable if and only if $\theta\left(V_{i, j}\right) \geq 0$ for any $i \in \bar{V}_{j}$ and $\theta\left(W_{i, j}\right) \geq 0$ for any $i \in \bar{W}_{j}$. It is $\theta$-stable if and only if the above conditions hold with strict inequalities.

Proof. The 'only if' direction is obvious. Let $U$ be a subrepresentation of $\mathcal{F}_{j}$. Without loss of generality, assume it is supported on the vertices $i, i+(r-a), \ldots, i+s(r-a)$. Then, by Definition 4.1, $i \in \bar{V}_{j}$ and $i+s(r-a) \in \bar{W}_{j}$ and hence $V_{i, j}, W_{i+s(r-a), j}$ are subrepresentations of $\mathcal{F}_{j}$. The lemma follows since $\theta(U)=\theta\left(V_{i, j}\right)+\theta\left(W_{i+s(r-a), j}\right)$.

EXAMPLE 7.4. The following diagram shows the representations $\mathcal{F}_{0}, \ldots, \mathcal{F}_{4}$, respectively, in the case of $\frac{1}{5}(1,2,3)$ (cf. Example 3.8). The solid arrows stand for arrows represented by a non-zero number, i.e., $\sigma_{j}$-distinguished.

$$
\begin{array}{ll}
\mathcal{F}_{0}: & 0 \stackrel{z_{0}}{\longrightarrow} 3 \stackrel{z_{3}}{\longrightarrow} 1 \stackrel{z_{1}}{\longrightarrow} 4 \stackrel{z_{4}}{\longrightarrow} 2 \\
\mathcal{F}_{1}: & 2 \stackrel{y_{0}}{\longleftarrow} 0 \stackrel{z_{0}}{\rightleftarrows} 3 \stackrel{z_{3}}{\longrightarrow} 1 \stackrel{z_{1}}{\longrightarrow} 4 \\
\mathcal{F}_{2}: & 1 \stackrel{z_{1}}{\longrightarrow} 4 \stackrel{y_{2}}{\longleftarrow} 2 \stackrel{y_{0}}{\stackrel{z_{0}}{\rightleftarrows}} 3 \\
\mathcal{F}_{3}: & 4 \stackrel{y_{2}}{\longleftarrow} 2 \stackrel{y_{0}}{\longleftarrow} 0 \stackrel{z_{0}}{\longrightarrow} 3 \stackrel{y_{1}}{\longleftarrow} 1 \\
\mathcal{F}_{4}: & 3 \stackrel{y_{1}}{\longleftarrow} 1 \stackrel{y_{4}}{\longleftarrow} 4 \stackrel{y_{2}}{\longleftarrow} 0
\end{array}
$$


Moreover, $\xi(5,2)(0)=2, \xi(5,2)(1)=4, \xi(5,2)(2)=3, \xi(5,2)(3)=1, \xi(5,2)(4)=0$ and, for example $\bar{V}_{2}=\{1,3,4\}, \bar{W}_{2}=\{2,3,4\}$. If $U$ is a subrepresentation of $\mathcal{F}_{2}$ consisting of vertices 2, 4, then $\theta(U)=\theta\left(V_{4,2}\right)+\theta\left(W_{2,2}\right)$.

DEFINITION 7.5. We set $\varphi(r, a)(j)=\langle\xi(r, a)(j)+(r-a)\rangle_{r}$ for any coprime $a, r$ and $j=0, \ldots, r-1$.

Note that since $\xi(r, a)$ is a permutation of the set $\{0, \ldots, r-1\}$, so is $\varphi(r, a)$. Let $n_{0}, \ldots, n_{r-1}$ be some rational numbers. The addition in the indices of $n_{i}$ 's is modulo $r$. Observe that there is a linear map $\boldsymbol{Q}^{r} \ni\left(n_{0}, \ldots, n_{r-1}\right) \rightarrow \mathrm{Wt}(Q)$ of rank $r-1$ sending $\left(n_{0}, \ldots, n_{r-1}\right)$ to the function $\theta \in \mathrm{Wt}(Q)$ such that $\theta(i)=n_{i}-n_{i+(r-a)}$ for $i=0, \ldots, r-1$ with kernel spanned by the vector $(1, \ldots, 1)$.

LEMMA 7.6. For any coprime $r$, $a$ and any rational numbers $n_{0}, \ldots, n_{r-1}$, set $\theta(i)=$ $n_{i}-n_{i+(r-a)}$. The representations $\mathcal{F}_{0}, \ldots, \mathcal{F}_{r-1}$ are simultaneously $\theta$-stable if and only if

$$
n_{\varphi(r, a)(0)}<n_{\varphi(r, a)(1)}<\cdots<n_{\varphi(r, a)(r-1)} .
$$

Proof. Fix $j$ and $i \neq \xi(r, a)(j)+(r-a)$. Then either the $z_{i-(r-a)}$ - or the $y_{i}$-arrow is $\sigma_{j}$-distinguished. In the first case, $i \in \bar{V}_{j}$ and $\theta\left(V_{i, j}\right)=n_{i}-n_{\xi(r, a)(j)+(r-a)}>0$. Otherwise $i-(r-a) \in \bar{W}_{j}$ and $\theta\left(W_{i-(r-a), j}\right)=-n_{i}+n_{\xi(r, a)(j)+(r-a)}>0$. By the definition of the permutation $\tau$ and by the definition of the divisors $Y_{i}, Z_{i}$, exactly $r-1-j$ of $z$-arrows are $\sigma_{j}$-distinguished. Moreover, if $y_{j^{\prime}}$-arrow is $\sigma_{j^{\prime}}$-distinguished, then it is $\sigma_{j}$-distinguished for any $j \geq j^{\prime}$ (cf. Lemma 6.5). To prove the 'only if' direction, note that if $\mathcal{F}_{0}$ is $\theta$-stable, then $n_{\varphi(r, a)(0)}$ is smallest in $n_{i}$ 's. If in addition $\mathcal{F}_{1}$ is $\theta$-stable, then $n_{\varphi(r, a)(1)}$ is second smallest, and so on. For the other direction, note that, for any $j=0, \ldots, r-1$, we have $\theta\left(V_{i, j}\right)>0$ for any $i \in \bar{V}_{j}$ and $\theta\left(W_{i, j}\right)>0$ for any $i \in \bar{W}_{j}$. By Lemma 7.3 the representation $\mathcal{F}_{j}$ is $\theta$-stable for $j=0, \ldots, r-1$.

REMARK 7.7. For any coprime $r$ and $a$, the set of stability conditions for which the representations $\mathcal{F}_{0}, \ldots, \mathcal{F}_{r-1}$ are simultaneously $\theta$-stable is a simplicial and top dimensional cone in the space $\operatorname{Wt}(Q)$, since it is in bijection with the cone given by the conditions $n_{\varphi(r, a)(0)}=0$ and $0<n_{\varphi(r, a)(1)}<\cdots<n_{\varphi(r, a)(r-1)}$.

8. Main theorem. We have defined a family of pairwise non-isomorphic representations of the McKay quiver $Q$ on the Danilov resolution $Y$, which are $\theta$-stable with respect to stability parameters $\theta$ determined in Lemma 7.6. The universal property of the moduli space $M_{\theta}(Q, R)$ will ensure that the Danilov resolution dominates one of its components.

Definition 8.1 (Craw, Maclagan, Thomas). Let $\theta \in \mathrm{Wt}(Q)$ be generic. The coherent component $Y_{\theta}$ is the unique irreducible component of the moduli $M_{\theta}(Q, R)$, containing representations of the McKay quiver with all arrows represented by a non-zero number (cf. [3, Theorem 4.3]).

Note that representations of the McKay quiver with all arrows represented by a nonzero number are $\theta$-stable under any stability condition $\theta \in \mathrm{Wt}(Q)$. The coherent component 
is reduced irreducible, not-necessarily-normal, toric variety of dimension 3, projective over $X=\boldsymbol{C}^{3} / G$ (see [3, Theorem 4.3]). Denote by $\pi_{\theta}$ the corresponding projective birational morphism

$$
\pi_{\theta}: Y_{\theta} \rightarrow X .
$$

Denote by $\pi$ the natural toric morphism given by a sequence of toric weighted blowups

$$
\pi: Y \rightarrow X,
$$

where $Y$ denotes the Danilov resolution.

Assume that the generic stability parameter $\theta$ is chosen so that all representations of the McKay quiver in the family $\mathcal{F}$ on $Y$ are $\theta$-stable. Since $\theta$ is generic, by King [12, Proposition 5.3], there exists a universal family of McKay quiver representations over $M_{\theta}(Q, R)$ and there exists a unique morphisms

$$
\rho: Y \rightarrow M_{\theta}(Q, R) .
$$

Since the family $\mathcal{F}$ is defined by Logvinenko's reductor condition, by [13, Theorem 4.1, Definition 1.4] we see that $\pi_{\theta} \circ \rho=\pi$.

THEOREM 8.2 (Main Theorem). For any coprime natural numbers $a, r$ and any rational numbers $n_{0}, \ldots, n_{r-1}$ such that

$$
n_{\varphi(r, a)(0)}<\cdots<n_{\varphi(r, a)(r-1)},
$$

where $\varphi(r, a)(j)=\langle\xi(r, a)(j)-a\rangle_{r}$ and $\xi(r, a)$ is the inverse of the permutation $\tau(r, a)$ (see Definition 3.1), the Danilov resolution of the singularity of type $\frac{1}{r}(1, a, r-a)$ is the normalization of the coherent component $Y_{\theta} \subset M_{\theta}(Q, R)$ for generic $\theta \in \mathrm{Wt}(Q)$, given by the condition

$$
\theta(i)=n_{i}-n_{i+(r-a)} .
$$

PROOF. Let $\theta$ be a generic stability condition satisfying the above conditions (cf. Remark 7.7). By Lemma 7.6, every representation of the McKay quiver in the family $\mathcal{F}(r, a)$ on the Danilov resolution, constructed in Section 5, is $\theta$-stable. Therefore, there exists a unique morphisms

$$
\rho: Y \rightarrow M_{\theta}(Q, R)
$$

and the following diagram commutes:

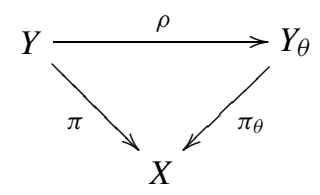

Morphism $\rho$ is proper since morphisms $\pi$ and $\pi_{\theta}$ are proper (see [7, Corollary II.4.8.(e)], $\pi_{\theta}$ is projective, hence separated). By [7, Exercise II.4.4], the image of $\rho$ in $Y_{\theta}$ is closed and is of dimension 3 (see Corollary 5.8).

By the work of Craw, Maclagan, Thomas [3], the coherent component $Y_{\theta}$ is a notnecessarily-normal toric variety of dimension 3 , hence $\rho$ is surjective onto $Y_{\theta}$. We are done 
if $\rho$ is injective, that is, representations of the McKay quiver corresponding to distinct closed points on the Danilov resolution are non-isomorphic. This is the content of Corollary 5.8.

DEFINITION 8.3. A chamber of stability conditions is a connected component of the set of generic stability conditions (cf. [5], [16]).

THEOREM 8.4. The closure of the cone defined by the condition of Theorem 8.2 is a union of closures of chambers of stability conditions for the action of the group $\operatorname{PGL}(Q, C)$ on the space $\operatorname{Rep}(Q, R)$.

ProOF. By proof of Lemma 7.6, if some of the inequalities in the condition for $n_{i}$ is not strict, then some representation $\mathcal{F}_{j}$ is strictly $\theta$-semistable. Conversely, if all inequalities are strict, then all representations $\mathcal{F}_{j}$ for $j=0, \ldots, r-1$ are $\theta$-stable.

We note that there may be non-generic points on the other components of $\operatorname{Rep}(Q, R)$ which may define walls subdividing the simplicial cone of stability conditions defined in Theorem 8.2.

EXAMPLE 8.5. The permutation $\tau(5,2)$ is cyclic of length 5 , namely

$$
\tau(5,2)=(0,4,1,3,2) .
$$

The sequence

$$
n_{0}<n_{2}<n_{1}<n_{4}<n_{3}
$$

implies that the union of closures of chambers is given by the conditions $\theta_{0}+\cdots+\theta_{4}=0$ and

$$
0 \leq \theta_{2} \leq \theta_{1}+\theta_{2}+\theta_{4} \leq \theta_{2}+\theta_{4} \leq-\theta_{0} .
$$

Using computer algebra packages, the author has checked that, for small values of $a$ and $r$ and stability parameters as in the Main Theorem, the moduli space of representation of the McKay quiver is normal. This suggests that the following holds.

CONJECTURe 8.6. The coherent component is normal in this case, that is, the Danilov resolution is isomorphic to the coherent component $Y_{\theta}$ for any $\theta$ in Theorem 8.2.

\section{REFERENCES}

[1] T. BRidgeland, A. KIng AND M. ReID, The McKay correspondence as an equivalence of derived categories, J. Amer. Math. Soc 14 (2001), 535-554.

[2] A. CRAW AND A. Is HII, Flops of $G$-Hilb and equivalences of derived categories by variation of GIT quotient, Duke Math. J. 124 (2004), 259-307.

[3] A. Craw, D. Maclagan and R. R. Thomas, Moduli of McKay quiver representations. I. The coherent component, Proc. Lond. Math. Soc. (3) 95 (2007), 179-198.

[ 4 ] V. I. DANILOv, Birational geometry of three-dimensional toric varieties, Izv. Akad. Nauk SSSR Ser. Mat. 46 (1982), 971-982, 1135.

[5] I. V. Dolgachev And Y. Hu, Variation of geometric invariant theory quotients, Inst. Hautes Études Sci. Publ. Math. 87(1998), 5-56. 
[ 6 ] W. Fulton, Introduction to toric varieties, Ann. of Math. Stud. 131, The William H. Roever Lectures in Geometry, Princeton University Press, Princeton, NJ, 1993.

[ 7 ] R. Hartshorne, Algebraic geometry, Graduate Texts in Mathematics, No. 52, Springer-Verlag, New York, 1977.

[ 8 ] Y. Ito AND H. NAKaJima, McKay correspondence and Hilbert schemes in dimension three, Topology 39 (2000), 1155-1191.

[ 9 ] Y. ITO AND I. NAKAmURA, Hilbert schemes and simple singularities, In New trends in algebraic geometry (Warwick, 1996), 151-233, London Math. Soc. Lecture Note Ser. 264, Cambridge Univ. Press, Cambridge, 1999.

[10] O. KĘDZIERSKI, Cohomology of the $G$-Hilbert scheme for $\frac{1}{r}(1,1, r-1)$, Serdica Math. J. 30 (2004), 293302.

[11] O. KęDZIERsKi, The G-Hilbert scheme for $\frac{1}{r}(1, \mathrm{a}, \mathrm{r}-\mathrm{a})$, Glasg. Math. J. 53 (2010), $115-129$.

[12] A. D. KING, Moduli of representations of finite-dimensional algebras, Quart. J. Math. Oxford Ser. (2) 45 (1994), 515-530.

[13] T. Logvinenko, Natural $G$-constellation families, Doc. Math. 13 (2008), 803-823.

[14] D. R. Morrison and G. Stevens, Terminal quotient singularities in dimensions three and four, Proc. Amer. Math. Soc. 90 (1984), 15-20.

[15] M. REID, Young person's guide to canonical singularities, Algebraic geometry, Bowdoin, 1985 (Brunswick, Maine, 1985), 345-414, Proc. Sympos. Pure Math. 46, Amer. Math. Soc., Providence, RI, 1987.

[16] M. ThadDEus, Geometric invariant theory and flips, J. Amer. Math. Soc. 9 (1996), 691-723.

INSTITUTE OF MATHEMATICS

WARSAW UNIVERSITY

UL. BANACHA 2

02-097 WARSZAWA

POLAND

E-mail address: oskar@mimuw.edu.pl 